\title{
A strategy for fitting partially blended ridges in GONG solar $p$-mode spectra
}

\author{
R. Howe ${ }^{\star}$ and M.J. Thompson
}

Astronomy Unit, School of Mathematical Sciences, Queen Mary and Westfield College, Mile End Road, London E1 4NS, UK e-mail: M.J.Thompson@qmw.ac.uk

Received October 1, 1997; accepted March 18, 1998

\begin{abstract}
Observations of solar oscillations by the Global Oscillation Network Group (GONG) using a network of telescopes deployed around the Earth eliminate temporal aliases from the oscillation power spectra to a very substantial extent, making the measurement of oscillation mode parameters much cleaner than with single-site observations. However, the problem of spatial aliasing, which arises from the fact that we see only one side of the Sun, still causes some problems. We discuss some of the issues affecting the estimation of medium- $l$ solar acoustic mode parameters from the data obtained by the GONG network, and in particular the contamination by spatial leakage. We determine the areas in the $l-\nu$ plane in which such leakage is a problem, and finally we describe a method for improving the mode frequency estimation in such regions, thus extending the range of modes for which useful frequency estimates can be obtained.
\end{abstract}

Key words: methods: data analysis — sun: oscillations

\section{Introduction}

The Sun oscillates simultaneously in many global normal modes. In helioseismology, we use in particular the frequencies of these modes to learn about the internal structure and dynamics of the Sun. The Global Oscillations Network Group (GONG) project (Harvey et al. 1996) has been obtaining good-quality, nearly continuous observations of a large number of these modes for nearly two years. The present paper discusses some of the problems that are encountered in deriving the frequencies from the raw data, and proposes a strategy for improving the treatment of one of these problems, the contamination of mode spectra by

Send offprint requests to: M.J. Thompson

* Present address: N.O.A.O., 950 N. Cherry Ave., P.O. Box 26732, Tucson, Arizona, 85726-6732, U.S.A. spatial leakage in ranges where the leaked power is not well resolved from the main mode power. We will first briefly outline the main steps involved in converting raw observations to frequency estimates, and introduce the problem of leakage between spectra. In the following sections, we will identify the regions where the leakage is a particular problem for the analysis, and finally present a strategy for working around the problem.

\section{Analysis overview}

The oscillations are detectable as periodic variations of line-of-sight velocity and intensity. Instruments such as those operated by the GONG network, or the helioseismology experiments on $\mathrm{SOHO}$, provide near-continuous series of images mapping e.g. the line-of-sight velocity of the solar surface. The raw velocity images can be described as

$S(\theta, \phi, t)=R e\left[\sum_{n l m} Y_{l}^{m}(\theta, \phi) s_{n l m}(t) \rho(\theta, \phi)\right]$

where $R e$ denotes the real part. Here $\theta$ and $\phi$ are the latitude and longitude on the solar surface, and $\rho(\theta, \phi) \equiv$ $\sqrt{1-r^{2}}$ is the term for the line-of-sight projection of the velocity on the visible disk, $r$ being the distance from disk centre. In real calculations, subtleties such as the variation in the so-called $B$-angle and apparent solar radius over the year have to be taken into account in the calculation of $r$.

The surface harmonic $Y_{l}^{m}$ reflects the horizontal structure of each normal mode, $Y_{l}^{m}=P_{l}^{m}(x) \exp (i m \phi)$, where $P_{l}^{m}(x)$ is an associated Legendre function, $x=\cos \theta$, and $l$ and $m$ are known as the degree and azimuthal order of the mode. If the oscillation of each mode proceeded uninterrupted and without growing or decaying, then each $s_{n l m}(t)$ would be just some complex constant times $\exp \left[i \omega_{n l m}(t)\right]$, where $\omega$ is the normal mode angular frequency. In practice, the stochastic excitation and decay of the modes over time mean that $s$ is a more complicated function, leading 
to finite mode lifetimes that depend on $\omega$ and to some extent on $l$.

These images are then processed to produce complex time series $S_{\text {obs }}(l, m, t)$, each corresponding to a spherical harmonic of different degree $l$ and azimuthal order $m$. This is achieved, effectively, by multiplying the image by the appropriate spherical harmonic $Y_{l}^{m}$ and in general also by a weighting or apodization function $A p(\theta, \phi)$ that gives less weighting to the edges of the image, and integrating the product over the most visible portion of the solar disk. The image is generally remapped at this stage onto a regular grid in $x=\cos \theta$ and $\phi$.

The time series in turn are Fourier-transformed to give power spectra. The existence of the normal modes of oscillation gives rise to peaks in these power spectra. Because of the finite lifetimes of the modes, and the stochastic nature of the excitation, the peaks have finite widths and a "ragged" appearance. Each spectrum contains the peaks corresponding to all radial orders $n$ for a given $l$ and $m$. As modes of different $n$ but the same $l$ and $m$ have different frequencies, however, these are easily separated.

In order to make inferences about solar structure and dynamics, it is necessary to estimate the mode parameters, and in particular the mode frequencies, as accurately as possible from the spectra. This is achieved by using some form of non-linear maximum-likelihood estimation to find the best fit of a model spectrum to the data. We discuss our own form of model fitting in Sect. 5. Generally, the model will consist of Lorentzian peaks superimposed on a flat or slowly-varying background, though other (perhaps asymmetric) peak profiles can be used.

\section{Spatial leakage}

One of the major problems in obtaining $p$-mode parameters from Fourier spectra such as those provided by the GONG network is that the separation into the different spherical harmonics is far from perfect; this is inevitable given that we observe only a portion of one side of the Sun. The $Y_{l}^{m}$ are by definition orthogonal over the whole solar surface, whereas we integrate only over a portion of the visible disk. Thus a spectrum for a given degree $l$ and azimuthal order $m$ will include contributions from other harmonics $\left(l^{\prime}, m^{\prime}\right)$ The size of the contributions to a time series for $(l, m)$ from harmonics $\left(l^{\prime}, m^{\prime}\right)$ can be expressed in terms of the factors $L_{l m l^{\prime} m^{\prime}}^{\mathrm{R}}$ and $L_{l m l^{\prime} m^{\prime}}^{\mathrm{I}}$ such that the observed time series for $(l, m)$, after apodization, will be

$$
\begin{aligned}
S_{\text {obs }}(l, m, t) & =\sum_{n^{\prime} l^{\prime} m^{\prime}} L_{l m l^{\prime} m^{\prime}}^{\mathrm{R}} \operatorname{Re}\left[s_{n^{\prime} l^{\prime} m^{\prime}}(t)\right] \\
& -i \sum_{n^{\prime} l^{\prime} m^{\prime}} L_{l m l^{\prime} m^{\prime}}^{\mathrm{I}} \operatorname{Im}\left[s_{n^{\prime} l^{\prime} m^{\prime}}(t)\right] ;
\end{aligned}
$$

here $I m$ denotes the imaginary part. The factors $L^{\mathrm{R}}$ and $L^{\mathrm{I}}$ are derived by considering the result of projecting the $\left(l^{\prime}, m^{\prime}\right)$ spherical harmonic on the $(l, m)$ mask and equating real and imaginary parts.

\subsection{Simplified leakage calculation}

In order to give some feel for how the main trends in the leakage come about, we present some expressions for the factors $L^{\mathrm{R}}$ and $L^{\mathrm{I}}$. These results were presented by Schou $\&$ Brown (1994) who give more details of their derivation.

Simplified expressions for the factors $L^{\mathrm{R}}$ and $L^{\mathrm{I}}$ are

$$
\begin{array}{rc}
L_{l m l^{\prime} m^{\prime}}^{\mathrm{R}} & =\frac{1}{\pi} \int_{-\frac{\pi}{2}}^{\frac{\pi}{2}} \int_{-1}^{1} P_{l}^{m}(x) P_{l^{\prime}}^{m^{\prime}}(x) \\
& \times \cos (m \phi) \cos \left(m^{\prime} \phi\right) A p(\theta, \phi) \rho(r) \mathrm{d} \phi \mathrm{d} x
\end{array}
$$

and

$$
\begin{array}{cc}
L_{l m l^{\prime} m^{\prime}}^{\mathrm{I}} & =\frac{1}{\pi} \int_{-\frac{\pi}{2}}^{\frac{\pi}{2}} \int_{-1}^{1} P_{l}^{m}(x) P_{l^{\prime}}^{m^{\prime}}(x) \\
& \times \sin (m \phi) \sin \left(m^{\prime} \phi\right) A p(\theta, \phi) \rho(r) \mathrm{d} \phi \mathrm{d} x .
\end{array}
$$

Certain simplifying assumptions are made in deriving these expressions. The horizontal component of the motion, which for the lowest order modes may make a significant contribution to the line-of-sight velocity, has been neglected. The effect of the finite pixel size of the detector, and hence the finite resolution of the observed image, has also been ignored. This effect becomes more important at higher $l$, but does not greatly affect the qualitative description of the leakage matrix. The finite Point Spread Function (PSF) is also ignored. Importantly, the visible area of the disk and the apodization have been assumed to be east-west symmetric (i.e. symmetric about $\phi=0$ ), and the $\theta=0$ axis has been assumed to lie in the plane of the sky. These assumptions mean that cross terms in $\sin (m \phi) \cos \left(m^{\prime} \phi\right)$ and $\cos (m \phi) \sin \left(m^{\prime} \phi\right)$ integrate to zero. All of these are reasonable assumptions to first order.

The contribution to the power spectrum is determined by the square of the so-called leakage element $L_{l m l^{\prime} m^{\prime}}$, defined by

$L_{l m l^{\prime} m^{\prime}} \equiv \frac{1}{2}\left(L_{l m l^{\prime} m^{\prime}}^{\mathrm{R}}+L_{l m l^{\prime} m^{\prime}}^{\mathrm{I}}\right)$.

It follows from Eqs. (3) and (4), and under the same assumptions, that

$$
\begin{gathered}
\quad=\int_{A} P_{l}^{m}(x) P_{l^{\prime}}^{m^{\prime}}(x) \\
\quad \times A p(\theta, \phi) \rho(r) \sin \theta \cos \left(\left(m-m^{\prime}\right) \phi\right) \mathrm{d} \theta \mathrm{d} \phi
\end{gathered}
$$

where the integral is over the area where $A p \neq 0$. Under the further assumption that the apodization is northsouth symmetric (which will be true, as will east-west symmetry, in the common situation where $A p$ is a function only of the distance from disk centre), the expression under the integral will be either odd or even in $\sin \psi$, where $\psi=\pi / 2-\theta$, depending on the parity of $\delta l+\delta m$. Here $\delta l=l-l^{\prime}$ and $\delta m=m-m^{\prime}$. Therefore, only leaks with $\delta l+\delta m$ even will contribute. In reality, there will be small contributions from asymmetric terms, and from the fact that the $P_{l}^{m}$ are not strictly orthogonal over the area of integration, but these are much less significant. 


\subsection{Some properties of the GONG leakage matrix}

Some examples serve to illustrate the important properties of the leakage matrix. The leakage has been calculated as realistically as we can for the GONG observations, using the real apodization function (the one used for the original processing of the first year or so of data) and taking the $\mathrm{B}$ angle into account when calculating the line-of-sight projection. We also take into account the subtraction of the $l=0$ time-series from the time series for $l>0$ that occurs in the real processing, but neglect the effects of finite pixel size and (as previously noted), the finite PSF.

First, though, in Fig. 1, we show schematically the major contributions to the region around an $(l, m)$ mode in the $(l, m)$ spectrum. The illustration is for an intermediate $l$ value, say $l=30$ to 150 . The separation between modes with the same $n$ and $l$, and $\delta m=2$, is typically around $0.8 \mu \mathrm{Hz}$ (here and henceforward, in keeping with the usual practice of observers, we work in terms of the cyclic frequency $\nu=\omega / 2 \pi)$, whereas the spacing $\partial \nu / \partial l$ between modes of the same $n$ and $m$ but with $\delta l=1$ will range from several $\mu \mathrm{Hz}$ to a few tens of $\mu \mathrm{Hz}$. In this regime $\partial \nu / \partial n$ will be large enough that we can ignore leaks from modes with different values of $n$ (see Sect. 4). The main peak, consisting of the $(l, m)$ mode and $(l, m \pm 2) m$-leaks which are (for frequencies greater than about $2 \mathrm{mHz}$ ) too close to be resolved from it, is flanked by ridges with $\delta l= \pm 1, \delta m= \pm 1$ and with $\delta l= \pm 2, \delta m=0, \pm 2$. For reasons of symmetry already discussed, leaks with $\delta l+\delta m$ odd do not appear.

Figure 2 illustrates the variation of the power of the $(l, m)$ mode in the $(l, m)$ spectrum as a function of $m$ for $l=50$. What is in fact plotted is the square $L_{l m l m}^{2}$ of the diagonal leakage matrix element. However, assuming that the mode has a physical amplitude equal to unity, this is equal to the power $P$. Note that the quantity plotted is not, in general, equal to 1 , due to attenuation caused by the line-of-sight effect and the apodization function. The near-zonal modes, which sample all latitudes, are attenuated more than the near-sectoral modes which are confined to a region around the equator.

When considering the power of leaked modes in a given spectrum $(l, m)$, the quantity of interest is often the ratio $R_{l m l^{\prime} m^{\prime}} \equiv L_{l m l^{\prime} m^{\prime}}^{2} / L_{l m l m}^{2}$ between the power of the leaked mode $\left(n^{\prime}, l^{\prime}, m^{\prime}\right)$ and the power of the target $(n, l, m)$ mode in the same spectrum, assuming that the physical amplitude of each mode is the same. For modes close together in $l$ and frequency, this will be approximately true. We thus refer to this quantity as $P_{\text {leak }} / P$, the ratio in the $(l, m)$ spectrum of the power of the spatially leaked $\left(l^{\prime}, m^{\prime}\right)$ mode to the power of the $(l, m)$ mode. In Figs. 3, 4 , and 5 we show the variation of the relative power of various leaks in the $(l, m)$ spectrum as a function of $m$ for an $l=50$ multiplet. (Refer to the schematic in Fig. 1 to see the typical alignment of leaks of different degree $l$ relative to the target mode.) Figure 3 shows the relative power in the leaks from modes with $\delta l=-1$; Fig. 4 shows the relative

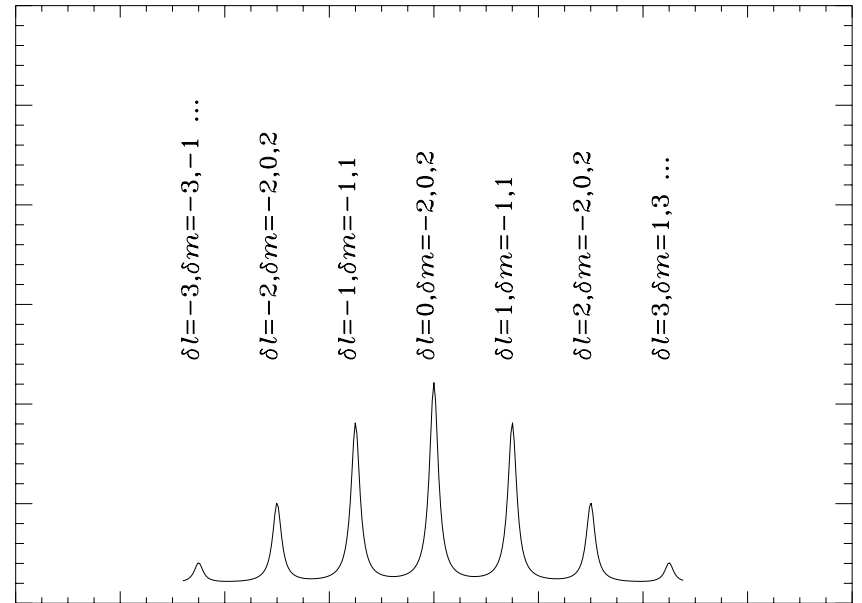

Fig. 1. Schematic plot of a section of the $(l, m)$ spectrum around an $(l, m)$ mode, showing the major leaked contributions to the power

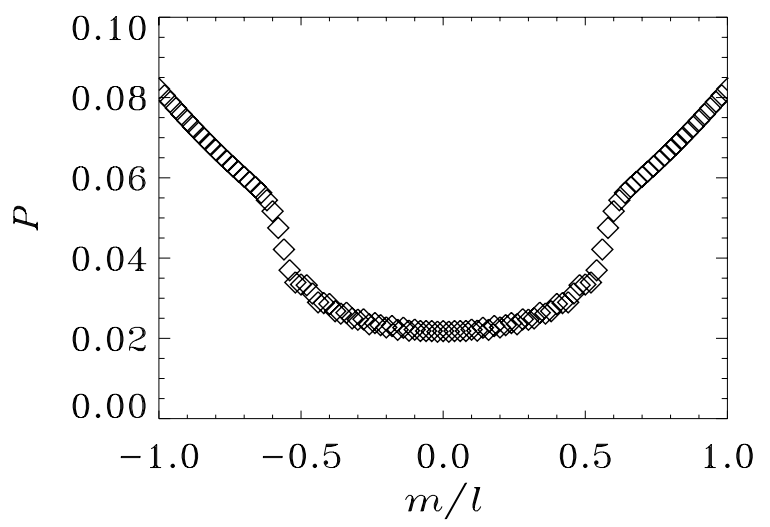

Fig. 2. Power of an $(l, m)$ mode in the $(l, m)$ spectrum (that is, $L_{l, m, l, m}^{2}$ ) for $l=50$

power in the leaks with $\delta l=-2$; while Fig. 5 shows the power in the generally unresolved modes that share the target $l$ (i.e. $\delta l=0$ ) but have $m$ differing from the target mode by 2 . One point of interest in these plots is that the ratio of the total power for the $(l, m)$ peak and its usually unresolved $(l, m \pm 2)$ leaks - essentially one plus twice the quantity plotted in Fig. 5 - to the total power in the $(l \pm 1, m \pm 1)$ leaks - i.e. the sum of the two contributions in Fig. 3 - is approximately independent of $m$. Thus the $\delta l=1$ ridge remains approximately the same height relative to the main ridge for all $m$. However, the changes in relative power in the various components of the leaks will result in a shift in the apparent frequency of the leaks as a function of $m$ : this could lead to systematic errors in the frequency determinations if the peaks were unresolved and asymmetric.

As Fig. 3 illustrates, for small $\delta l$ the leaked contributions can be almost as large as those from the target mode. This becomes a problem when the frequency separation between these leaks and the main peak becomes small; empirically, one cannot obtain reliable independent 


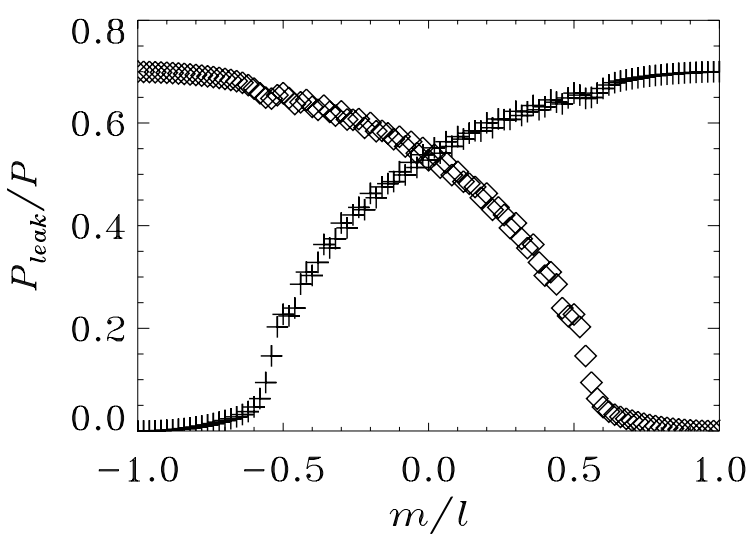

Fig. 3. Relative power of the $\delta l=-1, \delta m=-1$ (crosses) and $\delta l=-1, \delta m=+1$ (diamonds) leaks compared to the $(l, m)$ peak in the $(l, m)$ spectrum for $l=50$

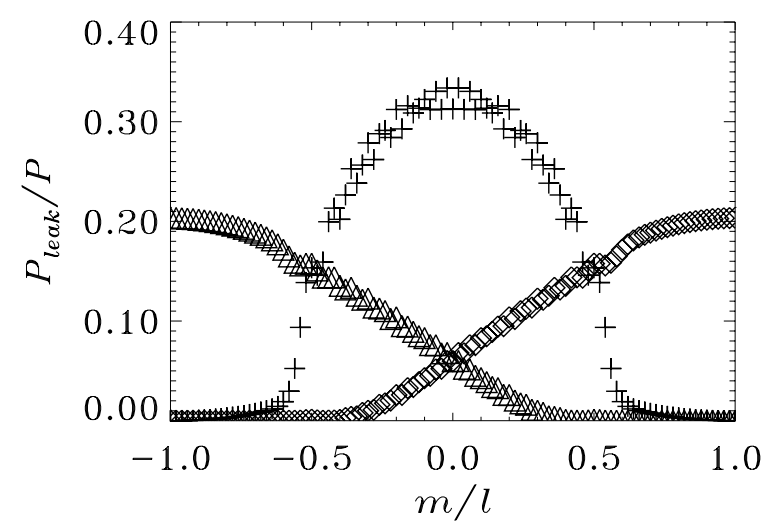

Fig. 4. Relative power of the $\delta l=-2, \delta m=-2$ (diamonds), $\delta m=0$ (crosses) and $\delta m=2$ (triangles) leaks compared to the $(l, m)$ peak in the $(l, m)$ spectrum, for $l=50$

estimates of the parameters (such as frequency, amplitude and linewidth) of two overlapping peaks if they are separated by a frequency difference $\Delta \nu$ less than twice their linewidth $\Gamma$ - defined to be their full width at half maximum height (FWHM). This criterion is rather stricter than the usual Rayleigh criterion requiring peaks to be one linewidth apart to be resolved. This is due partly to the broad wings of the peaks, which follow an approximately Lorentzian profile, and partly to the nature of the multiplicative noise.

This simple criterion using the ratio $\Delta \nu / \Gamma$ can be used to identify ranges of $l$ and frequency $\nu$ in the solar $p$-mode spectrum where the target modes are well separated from their associated spatial leaks, and the regions where they are not. This provides an indication of the ranges of $l$ and $\nu$ over which some knowledge of the leakage matrix is likely to be necessary in order to estimate the mode parameters. As we shall show, there are particular problems at high frequencies for all degrees, and at high degree.

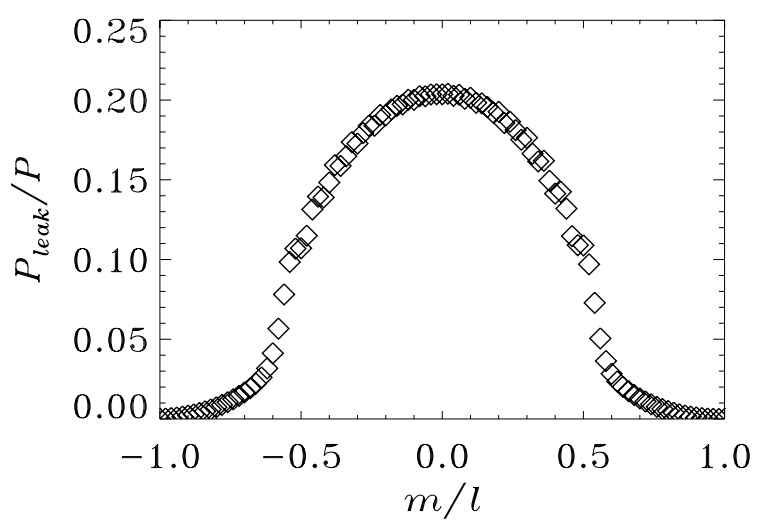

Fig. 5. Variation of $\delta m=2$ leak power relative to $(l, m)$ peak in the $(l, m)$ spectrum for $l=50$

\section{Peak spacing and linewidth in the $l-\nu$ plane}

The $p$-mode spectrum can conveniently be represented by a plot in the $l-\nu$ plane in which each $(n, l)$ multiplet is represented by a point corresponding to its central frequency. In such a diagram, modes of the same $n$ lie along clearly-defined "ridges". In order to locate the regions of the $l-\nu$ plane where modes are likely not to be wellseparated from leaks, we need to know how the linewidth $\Gamma$ varies as a function of $\nu$ and $l$.

We illustrate in Fig. 6 the mode linewidths as observed by GONG. The linewidths have been estimated by a fitting procedure, as briefly described by Hill et al. (1996). We have then fitted to these estimated linewidths $\Gamma$, averaged over $m$, a sum of two cubic splines in $\nu$ and $l$. This provides a smooth approximation to the linewidth, which is more useful for illustrating and mapping the general trends in the $l-\nu$ plane than the necessarily noisy estimates for individual modes. The GONG estimates are unfortunately available only up to $l=150$, because the fitting technique used is not appropriate when the ridges become blended. To fit to higher l's we need to use the leakage matrix in some way, for example as is already done for the MDI data or as described here. It is worth commenting briefly on the linewidths shown in this figure. Panel (a) shows a strong general trend for the linewidth to increase with frequency, although with a plateau between about $2200 \mu \mathrm{Hz}$ and $3000 \mu \mathrm{Hz}$. This behaviour is well established observationally, as reported by, for example, Elsworth et al. (1988), Libbrecht (1988) and Jefferies et al. (1991), and can be approximately reproduced in theoretical calculations of damped, stochastically excited oscillations (e.g. those of Balmforth \& Gough 1990 which take into account the damping associated with the coupling to convection). It is interesting that the observations indicate that the linewidth actually decreases slightly with frequency along the plateau, a feature remarked on by Balmforth \& Gough and present also in their theoretical calculations. Panel (b) shows the linewidths as a function of $l$, over a narrow range of frequency where the frequency 

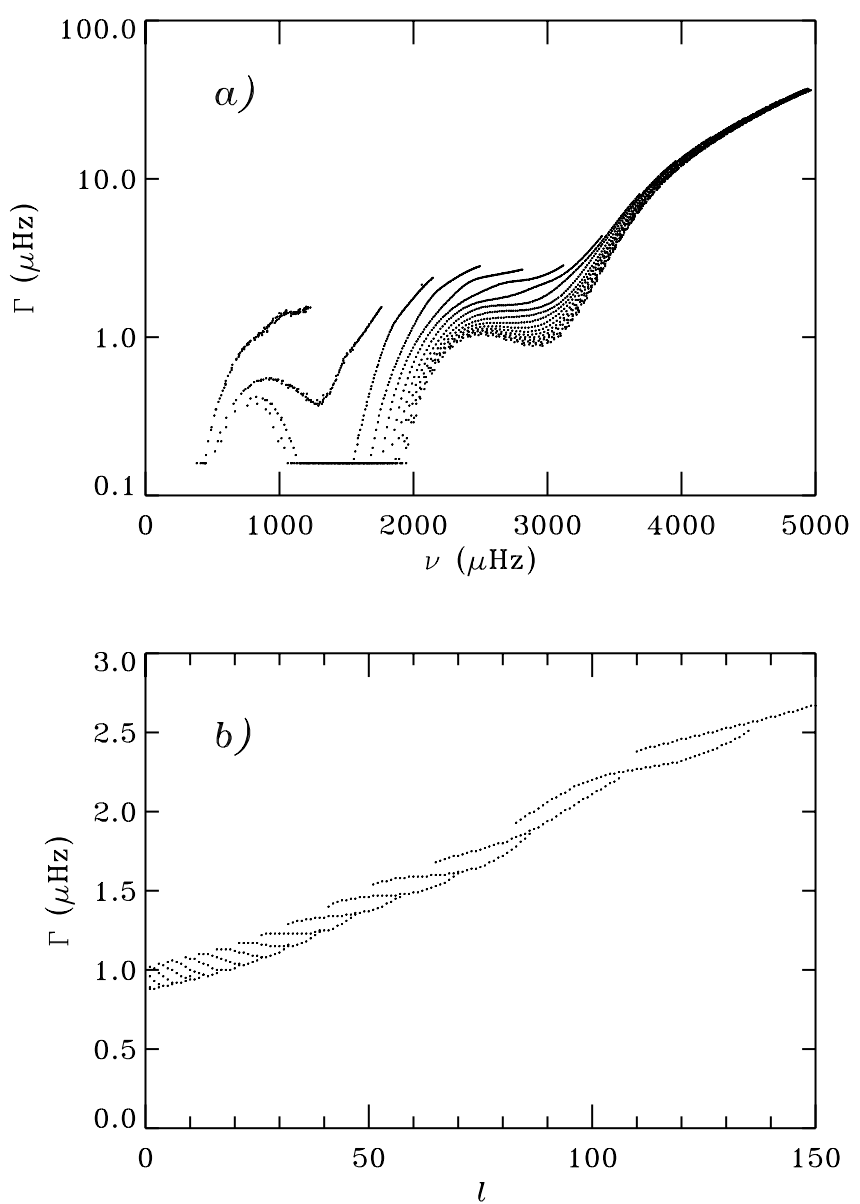

Fig. 6. The approximated linewidth $\Gamma$ a) as a function of frequency $\nu$, and $\mathbf{b}$ ) as a function of $l$ for the frequency range $2500 \leq \nu \leq 3000 \mu \mathrm{Hz}$ (where the width depends only weakly on $\nu$ ). The fit to $\Gamma$ is not allowed to be smaller than half a frequency resolution bin-width, i.e. $0.32 \mu \mathrm{Hz}$ for the time series used in this plot

dependence is relatively weak. This brings out the weaker but clearly discernible trend for the linewidth to increase with degree, consistent with the observations of Jefferies et al. (1991). The ridges of nearly connected dots in this figure correspond to modes of like $n$. The modes at the low-frequency end of these ridges are at higher values than one would get if one were to put a smooth fit through the high-frequency ends: this is because the figure is over a narrow range $2500 \mu \mathrm{Hz}<\nu<3000 \mu \mathrm{Hz}$ where in fact the linewidth decreases slightly with increasing frequency.

As we shall show, except at low $l$ the closest significant leaks are from modes of the same $n$ but with $\delta l= \pm 1$. As illustrated in Fig. 5, leaks of the same $l$ and $n$, with $\delta m= \pm 2$, have no more than 25 to 30 per cent of the main peak power. In general these leaks have similar relative power on either side of the main peak where $|\mathrm{m} / \mathrm{l}|$ is small, and are weak where $|m / l|$ is large. Ideally these " $m$-leaks" ought to be taken into account in fitting, but they are usually neglected. To see how the leaks affect the analysis of power spectra, we need to examine individual

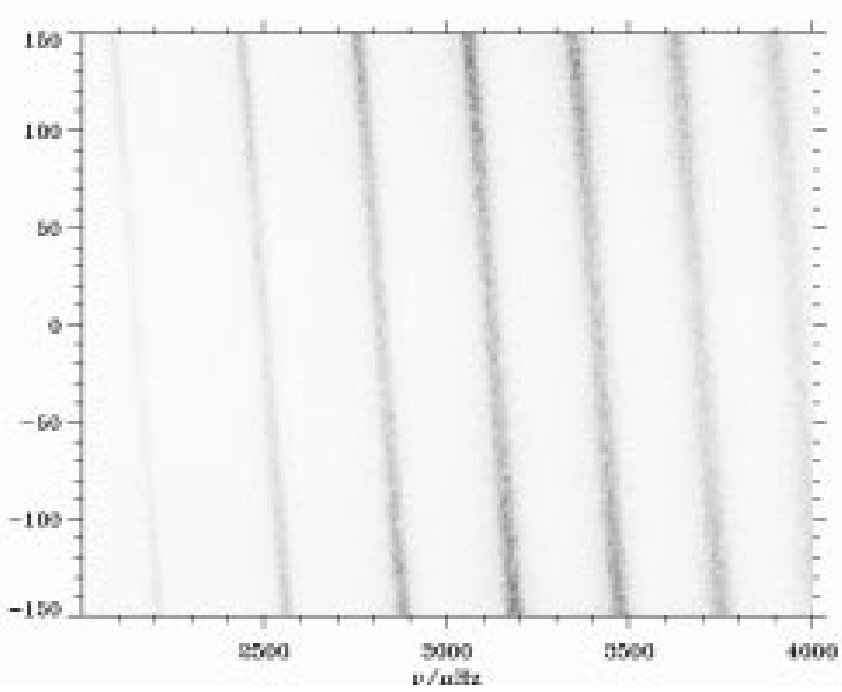

Fig. 7. A greyscale plot showing a portion of the GONG month 4 power spectrum for $l=150$ in the $m-\nu$ plane

spectra or " $m-\nu$ " diagrams that map the power in spectra of a given $l$ as a function of $m$ and frequency. Plotting the data for high $l$ spectra in this way reveals that each "ridge" of power at a given $l$ and $n$ is flanked by weaker ridges of power leaked from spectra of adjacent $l$, as illustrated in Fig. 7. Further examples of spectra and $m-\nu$ diagrams can be seen in Hill et al. (1996). The solar rotation shifts peaks of different $m$ away from the central frequency. Roughly speaking, the slope of the $(l, n)$ ridges in $m-\nu$ space reflects the mean rotation rate; the slight S-shaped curvature of the ridges arises from the differential rotation, which provides important information about the variation of the rotation rate with latitude and depth. In order to derive a useful " $m$-averaged spectrum" we first need to remove the $m$-dependence of the mode frequencies.

We can write the difference in frequency between modes that have the same value of $n$ but degrees $l$ differing by 1 as a derivative $(\partial \nu / \partial l)_{n}$, although $\nu(n, l)$ is not strictly speaking a continuous function.

Contours of $\Gamma^{-1} \partial \nu / \partial l$ are shown in Fig. 8. We anticipate particular difficulty in fitting peaks independently when this ratio is less than 2 . This occurs at high frequency (around $3500-4000 \mu \mathrm{Hz}$ ), because the linewidths become large, and also at high degree $(l \gtrsim 150)$ where $\partial \nu / \partial l$ becomes small. The latter tendency can be seen from the approximate formula for medium and high $l$,

$\omega^{2}=g k(n+\alpha)$

(e.g. Deubner \& Gough 1984), where $k=l / R, g$ is the gravitational acceleration, $R$ is the solar radius and $\alpha$ is a constant of order unity. From this formula,

$\left(\frac{\partial \nu}{\partial l}\right)_{n} \simeq \frac{\nu}{2 l}, \quad\left(\frac{\partial \nu}{\partial n}\right)_{l} \simeq \frac{\nu}{2(n+\alpha)}$

assuming that $g$ is constant. This indicates that at fixed $\nu$, the spacing $\partial \nu / \partial l$ decreases with $l$. (In fact the effective 


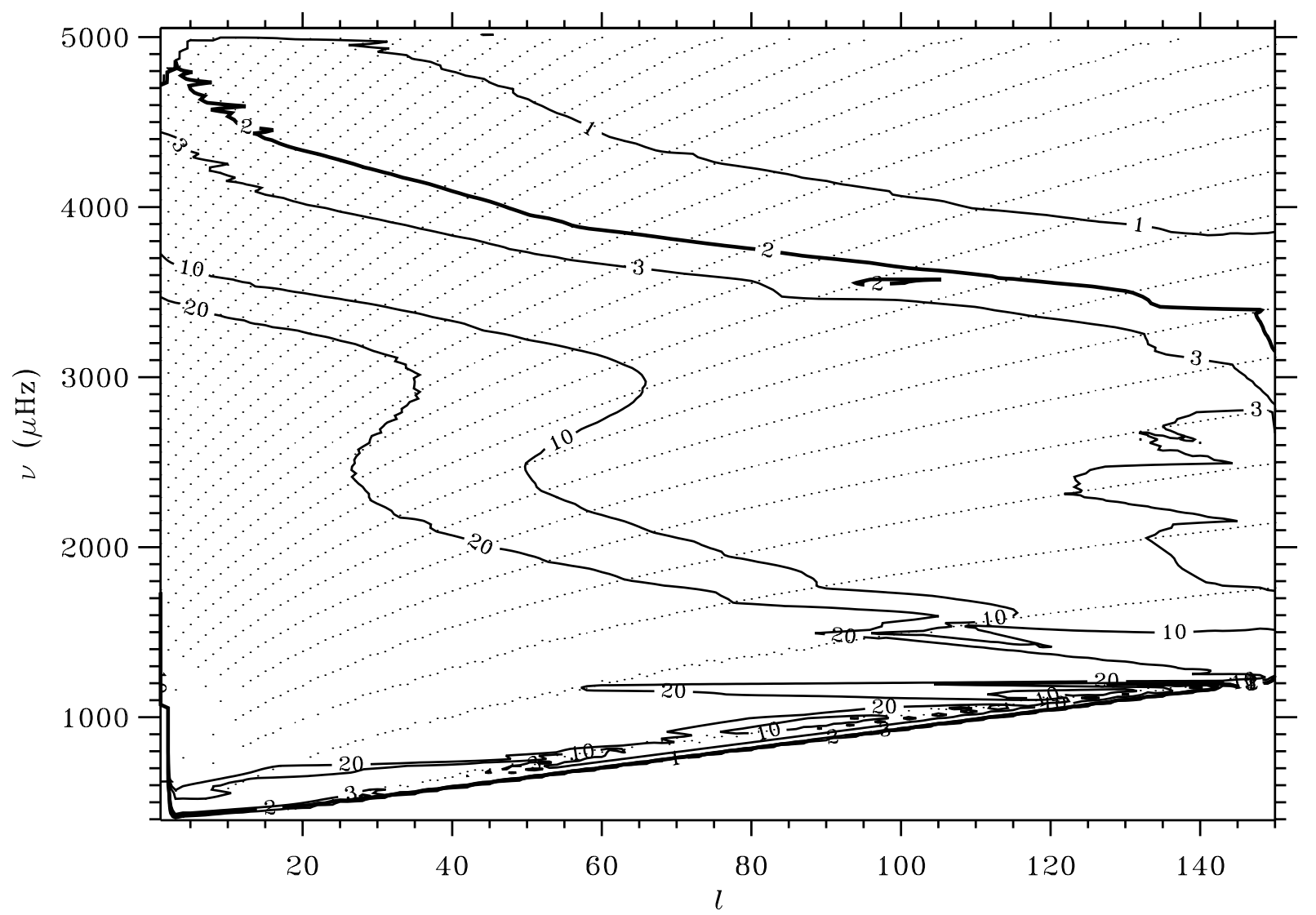

Fig. 8. An $l-\nu$ diagram showing contours of $(\partial \nu / \partial l) / \Gamma$. Each dot represents the mean position of an $(n, l)$ multiplet, each ridge of dots corresponding to a single value of $n$. Fitting of modes becomes substantially more difficult outside the $(\partial \nu / \partial l) / \Gamma=2$ contour, which is shown as bold

gravitational acceleration increases with decreasing turning point radius for modes of medium and high degree because going down through the convection zone the interior mass decreases more slowly than $r^{-2}$ increases - so the above formulae overestimate $\partial \nu / \partial l$ and underestimate $\partial \nu / \partial n$, though they are good for order-of-magnitude and qualitative behaviour.) The above approximation implies $(\partial \nu / \partial n) /(\partial \nu / \partial l) \simeq l /(n+\alpha)$. Since $\alpha$ is of order unity, it follows that at moderate and high degree (where $l \gg n$ ) the frequency spacing between neighbouring $n$ 's is much greater than between neighbouring $l$ 's. The ratio decreases however as $l$ decreases, so that at low degree it is necessary to be concerned also with nearby leaks from modes of different order $n$. At the lowest degrees, a more appropriate approximation for the frequencies is the Tassoul formula $\nu \simeq(n+l / 2+\epsilon) \nu_{0}$ where $\nu_{0} \approx 140 \mu \mathrm{Hz}$ and $\epsilon$ are constants, whence it can be seen that

$(\partial \nu / \partial n) /(\partial \nu / \partial l) \simeq 2$.

In the case where $(\partial \nu / \partial n) /(\partial \nu / \partial l) \leq \Delta l(\Delta l=2,3, \cdots)$, one needs to be aware of the possibility of " $n$-leaks" neighbouring modes with different radial order $n$ - for $|\delta l| \leq \Delta l$. Where $(\partial \nu / \partial n) /(\partial \nu / \partial l)$ is very close to $\Delta l$, leaks with $\delta l= \pm \Delta l$ will be particularly problematic. In Fig. 9 we show the modes whose nearest neighbour is within two linewidths: different symbols are used to show the modes affected by particular leaks. In addition to all the modes with $\delta \nu / \delta l<2 \Gamma$ (crosses), for which the nearest significant leak generally has $l$ differing from the target degree by one, this figure indicates that there are difficulties at moderately low degree due to leakage from modes with different $n$ (and hence - to have similar frequency to the target mode - with degrees $l$ differing from the target degree by more than one). Note that the leakage decreases fairly quickly with $|\delta l|$, and is typically less than one per cent of the mode power at $|\delta l|=5$. Thus leaks from modes with substantially different values of $l$ are not a principal concern.

\section{A template-fitting procedure}

We deduce from the above considerations that there are substantial regions of the $l-\nu$ plane where attempts to fit independently all the peaks in a given frequency range of a given $(l, m)$ spectrum are likely to fail; indeed, the region where such fitting is appropriate is a relatively small fraction of the full $l-\nu$ plane that is accessible to measurements from GONG or the MDI instrument on SOHO. To make inferences about mode parameters outside this 


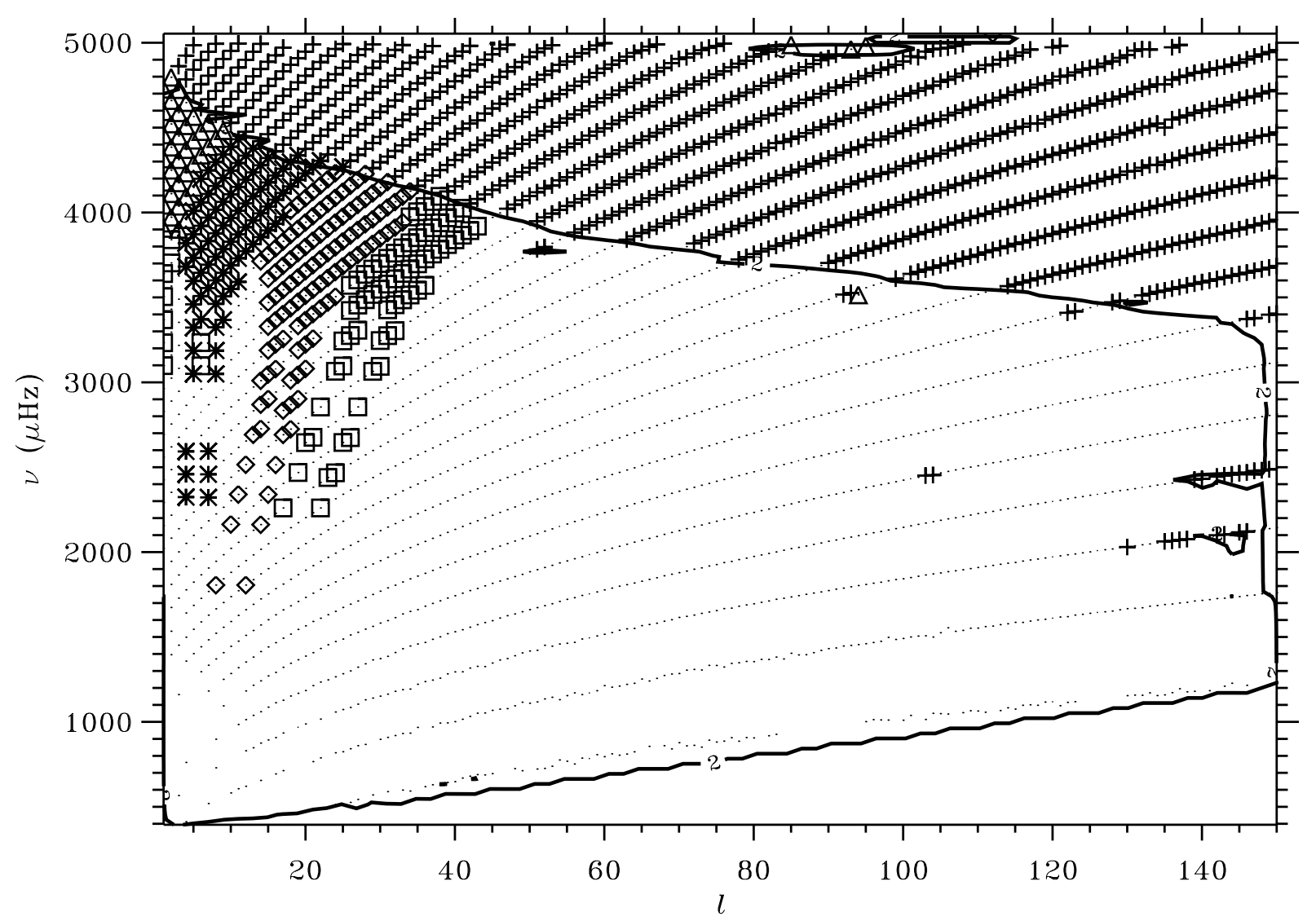

Fig. 9. An $l-\nu$ diagram illustrating the regions where $n$-leaks are a potential problem. The different symbols show modes affected by leaks within $2 \Gamma$ for various $\delta l$ : $\delta l= \pm 1$ for crosses, $\delta l= \pm 2$ for triangles, $\delta l= \pm 3$ for stars, $\delta l= \pm 4$ for diamonds and $\delta l= \pm 5$ for squares. The curve shows the $(\partial \nu / \partial l)=2 \Gamma$ contour

region, we need to make some assumptions about the position and relative strength of the leaked modes in the spectrum. However, the proper calculation of the leakage matrix at high degree is computationally expensive. The approach to mode-fitting described here aims to work around this by fixing some of the parameters of the fit in advance, using information obtained from the data rather than a detailed leakage model.

To illustrate the procedure, we shall concentrate on one typical multiplet, that with $l=150, n=4$ in GONG month 4 .

The first stage in the process is to locate the target system of peaks and leaks, which forms one of the broad, slanted bands of power shown in the greyscale plot in Fig. 7. This requires only approximate knowledge of the $m=0$ frequency of the target mode and its nearest neighbouring leaks $(\delta l= \pm 1)$, and the linear part of the $m$-dependence arising from rotation. Figure 10 shows the power in the region of interest in the $m-\nu$ plane with the linear part of the rotational dependence taken out to show the differential rotation more clearly. The data are averaged over bands covering several $m$-values, and smoothed in frequency, to clarify the structure. Next we locate the maximum of the smoothed data in each band as shown in the figure.

A better approximation to the $m$-dependence due to rotation can be obtained from a third-order polynomial fit to the position of the smoothed maxima as a function of $m$. Then, taking this $m$-dependence into account, the individual $m$ spectra can be collapsed to form a first approximation to the $m$-averaged spectrum.

Under standard assumptions that the modes are randomly and independently excited with a white-noise forcing function, at each frequency point the real and imaginary parts of the Fourier transform of the individual- $m$ time series $S_{\text {obs }}$ will be normally distributed with zero mean. (This is true even when leakage is taken into account, since the sum of independent Normal variables is itself Normal.) Thus at each frequency point the individual$m$ power spectra are proportional to a chi-squared variable with two degrees of freedom. (Of course, as discussed in more detail by Appourchaux et al. 1998, in the presence of leakage the different individual- $m$ spectra will not in general be independent.)

Since there are $2 l+1$ individual $m$ spectra for a given $l$, and notwithstanding the fact that the errors in each spectrum may not be entirely independent, we assume 


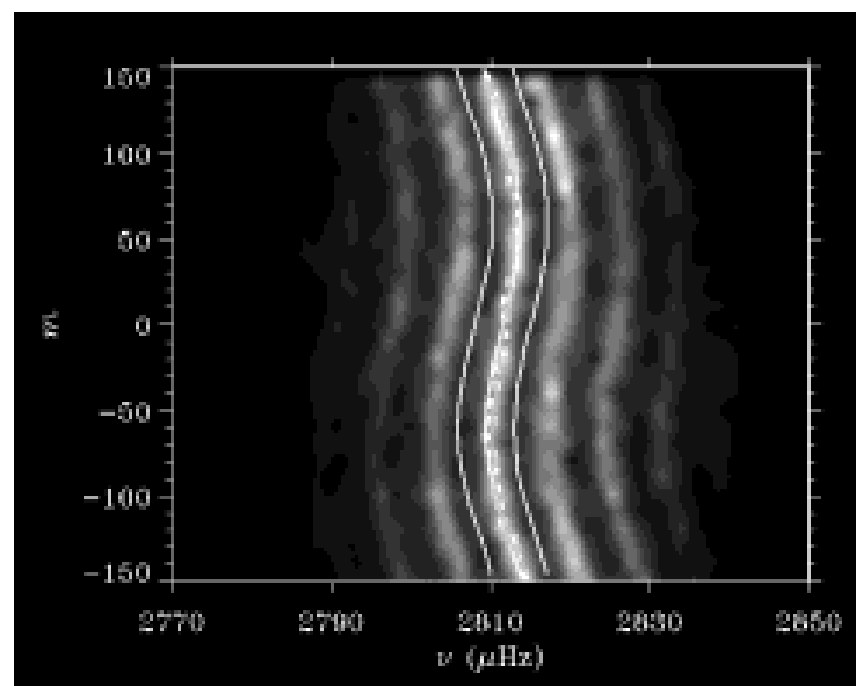

Fig. 10. Smoothed $m-\nu$ power spectrum of the $n=4, l=150$ mode and its leaks. The linear part of the $m$-dependence of the frequencies has been removed. The stars indicate maxima of the central ridge, and the curve shows a polynomial fit through these maximum points. The parallel curves indicate the frequency range, determined from the initial estimate of the linewidth, in which the maximum is sought

that by the Central Limit Theorem the errors in the $m$ averaged spectrum for high $l$ approach a normal distribution, and we proceed to fit the spectrum using a nonlinear least-squares method in which the variance at each frequency point is approximated as being proportional to the data value divided by the number of spectra averaged. The algorithm used is based on the CURFIT routine of Bevington (1969). The model consists of seven peaks - the target and the leaks out to $\delta l= \pm 3$ - of equal width, evenly spaced and with the relative powers of the leaks forced to be symmetrical, on a flat background, as illustrated in Fig. 11. The peak profile is assumed to be Lorentzian, defined by

$P\left(\nu, \nu_{0}, P_{0}, \Gamma\right)=\frac{(\Gamma / 2)^{2} P_{0}}{\left(\nu-\nu_{0}\right)^{2}+(\Gamma / 2)^{2}}$

where $\nu_{0}$ is the central frequency of the peak, $\Gamma$ the FWHM and $P_{0}$ the maximum height of the peak; $\nu$ is frequency. The full model for the $m$-averaged spectrum is thus given by

$T\left(\nu, \nu_{0}\right)=\sum_{\delta l=-3}^{3} P\left[\nu, \nu_{0}-\delta l \times \delta \nu, P_{0}(|\delta l|), \Gamma\right]+B_{0}$,

where $\delta \nu$ is the spacing (assumed uniform) between the peaks, $B_{0}$ is the background level of the power, and $P_{0}(|\delta l|)$ is the power of each ridge, which in this symmetric model depends only on $|\delta l|$. Thus we determine eight constants $-\nu_{0}, \Gamma, \delta \nu, B_{0}$ and four amplitudes $P_{0}(0,1,2,3)$ - which together define the fitted model $T\left(\nu, \nu_{0}\right)$.

This $T\left(\nu, \nu_{0}\right)$, which has been obtained by fitting the $m$-averaged spectrum, then becomes the "template" used

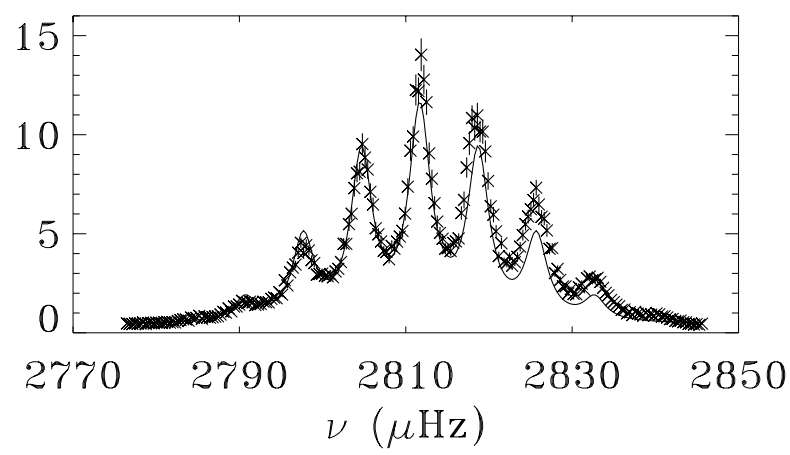

Fig. 11. The $m$-averaged spectrum of the $l=150, n=4$ mode (crosses) after removal of a third-order polynomial approximation to the $m$-dependence due to rotation. The continuous curve shows the fit of a symmetrical model comprising 7 equally spaced Lorentzian peaks. The units of power are arbitrary

to fit to the individual- $m$ spectra. To each $m$ spectrum we fit a model,

$P(\nu, m)=A_{\mathrm{T}}(m) T\left[\nu, \nu_{\mathrm{T}}(m)\right]+B_{\mathrm{T}}(m)$,

with the vertical scale $A_{\mathrm{T}}(m)$, background level $B_{\mathrm{T}}(m)$ and central frequency $\nu_{\mathrm{T}}(m)$ as the only free parameters. As already stated, we assume that each point of the spectrum is proportional to a chi-squared random variable with two degrees of freedom. The fit is then obtained by minimizing

$M=\sum_{i=1}^{n_{\mathrm{p}}} \log y_{\mathrm{m}}\left(\nu_{i}\right)+y_{i} / 2 y_{\mathrm{m}}\left(\nu_{i}\right)$,

where the index $i$ runs over the number $n_{\mathrm{p}}$ of data points in the fitting region (which extends roughly 10 times $\delta \nu$ either side of the central frequency), $\nu_{i}$ and $y_{i}$ are the values of the frequency and the power at each point in the fitting region, and $y_{\mathrm{m}}(\nu)$ is the model function. This is equivalent to maximizing the likelihood function, given the assumed chi-squared statistics. (See Anderson et al. 1990, for a discussion of this kind of fitting.) Any reasonable minimization technique can be used; in the example followed here, we used an IDL version of the DFPMIN routine from Press et al. (1989).

From these crude fits we obtain an improved approximation to the $m$-dependence of the splitting $\delta \nu(n, l, m)$, defined by

$\delta \nu(n, l, m)=\frac{1}{2}(\nu(n, l,-m)-\nu(n, l, m))$,

which is sufficient to provide a starting point for the iteration of more realistic fits. We next approximate the splitting as a function of $m$ with a sum of Legendre polynomials $P_{i}(m / L)$, where $L=l+1 / 2$, containing $n_{\mathrm{a}}$ odd and even terms:

$\delta \nu_{\text {model }}(n, l, m) / L=\sum_{i=0}^{n_{\mathrm{a}}} a_{i}(n, l) P_{i}(m / L)$.

This expression for $\delta \nu$ is used in turn to recompute the collapse of the individual $m$ spectra and yields an improved $m$-averaged spectrum. 
In the next iteration, the template is obtained by fitting the $m$-averaged spectrum with a model that allows the leaks to have independent width, relative power and spacing, so that the template is given by

$T(\nu, m)=\sum_{\delta l=-3}^{3} P\left[\nu, \nu_{0}-\delta \nu(\delta l), P_{0}(\delta l), \Gamma(\delta l)\right]+B_{0}$,

where $\delta \nu(\delta l)$ is now the separation of each ridge from the central frequency (for $\delta l \neq 0$ ). Thus we now determine a central frequency $\nu_{0}$, six constants $\delta \nu$, seven constants $P_{0}$, seven constants $\Gamma$ and one background. Furthermore, the fitting is done separately over three ranges of $m$, to allow some variation with $m$ of the leak power and spacing. This is illustrated in Fig. 12. The existence of such variation can be deduced even from a simplified version of the leakage calculation. The spacing variation arises because each leaked "peak" is really a doublet with $\delta m= \pm 1$ peaks, separated by about $2 a_{1}$, where $a_{1}$ is the leading rotational splitting coefficient as in Eq. (15). The strength of the two leaks varies, as illustrated in Fig. 3 ; and so as $m$ goes from $-l$ to $l$ the main contribution to the power in the ridge shifts from the component that is farther from the target $(l, m)$ to the one that is closer. Similar arguments apply to the leaks with $|\delta l|>1$, though these leaks are triplets rather than doublets. Dividing the fits in this way does not appear to introduce any noticeable discontinuities in the results; the division was chosen after consideration both of the simple leakage model behaviour discussed above and the behaviour of the real data.

Each template is then used as before over the appropriate range of $m$ to obtain the final frequencies. Figure 13 shows the final splittings derived for our multiplet, again with the linear $m$-dependence removed to show the curvature.

\section{Discussion}

One obvious limitation of the template method is that, as several leaks are included in each fit and their relative frequencies are not free parameters, the frequencies obtained at each $l$ will not be completely independent of those for adjacent degrees. The method is also susceptible to variations in the relative power of the leaks, and does not make use of the leak information in fitting other spectra where the leak might be a target mode. However, a method of this kind may be useful for extending fitting into regions where the modes are only partly resolved from the leaks. This kind of technique is potentially applicable to data from any instrument which is sensitive to sufficiently high degrees that blending becomes problematic. It might be useful for data from SOI-MDI on board SOHO, for example. In tests, we obtained reasonably satisfactory results up to about $l=190$ or $\partial \nu / \partial l=1.3 \Gamma$. In Fig. 14 we show the $m$-averaged spectrum for the $l=150, n=7$ multiplet, which illustrates the difficulty when the leak ridges come
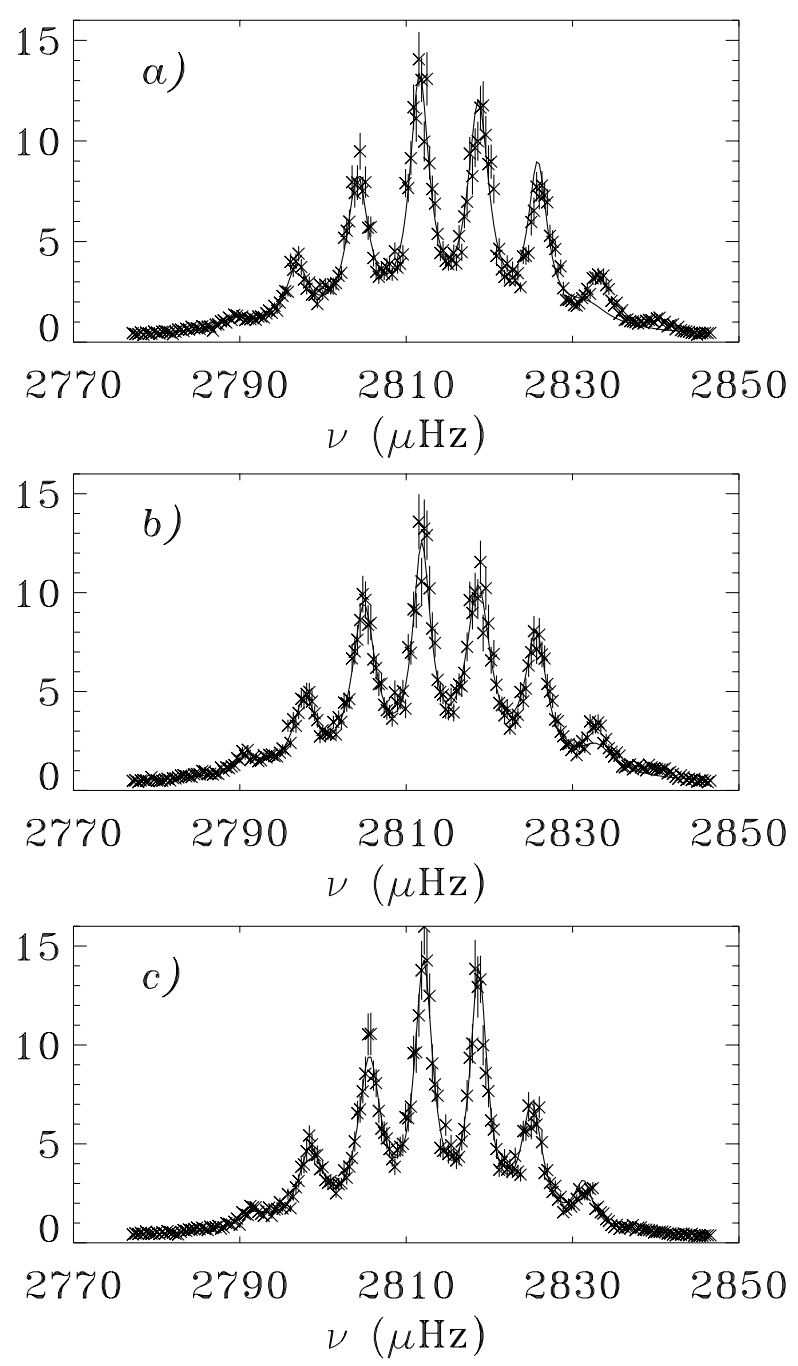

Fig. 12. The $m$-averaged spectra (symbols) for the $l=150, n=$ 4 mode, showing fits (solid curve) to a seven-peak model where all peaks have independent parameters, for a) $-150 \leq m \leq$ -50 , b) $-50<m \leq 50$, and c) $50<m \leq 150$

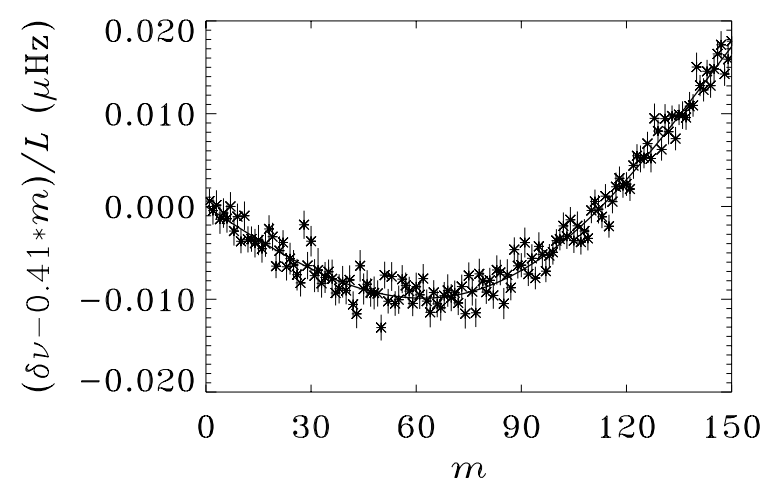

Fig. 13. Splittings $\delta \nu$, as defined in Eq. (14), for the $l=150, n=$ 4 multiplet, with linear $m$-dependence removed. The solid curve shows the fitted $\delta \nu_{\text {model }}$ as defined in Eq. (15) 


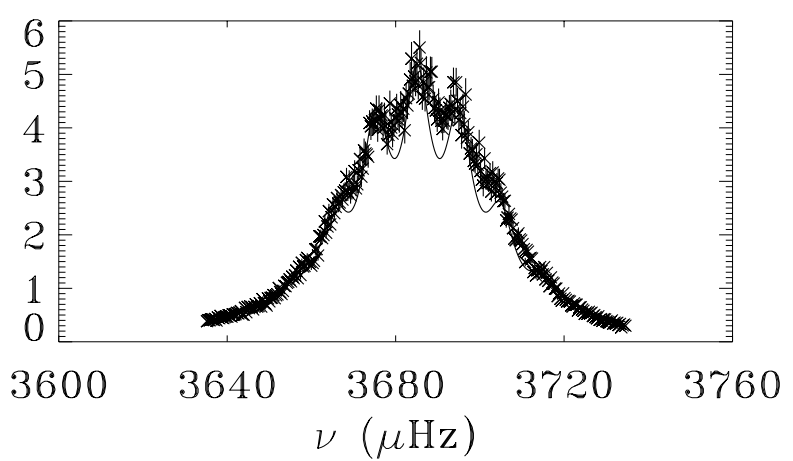

Fig. 14. The $m$-averaged spectrum (symbols) for the $l=$ $150, n=7$ multiplet, with the attempted fit to a symmetric seven-peak model shown as the curve. This is not considered a good fit

too close to the main one. This fit would probably have been rejected if we were applying the fitting scheme in a systematic manner.

Fitting the leaked ridges as single Lorentzian profiles is not strictly correct; for example, as noted above, each " $\delta l= \pm 1$ leak" consists of two peaks with $\delta m= \pm 1$, and the " $\delta l=2$ ridges" have three components with $\delta m=$ $-2,0$, and 2 , with relative strengths varying with $m$.

We have also ignored the asymmetry of the ridges in the data which, though clearly visible in the $m$-averaged spectra and $m-\nu$ diagrams, is not accounted for by the leakage-matrix calculations. Some of this asymmetry, which can be seen for instance in Fig. 12, is believed to arise from the neglected horizontal components of the motion in these low-order modes.

It should also be noted that a symmetrical Lorentzian profile may not be an adequate model for an individual peak because of the asymmetry introduced by the location of the excitation sources for the modes, as discussed, for example, by Abrams \& Kumar (1996) and by Nigam et al. (1998).

At higher degrees, the modes lose their global nature and the peaks for a given $l$ become completely blended with their adjacent leaks. Under these conditions, the approach described above breaks down because the peaks in the template cannot be independently fitted even in the $m$-averaged spectrum. "Ridge fitting" of a single unresolved peak at each $m$ will give reliable results only if the shape of this compound peak accurately reflects the relative strengths of the unresolved components. To achieve this, knowledge of the leakage matrix would almost certainly be necessary.

Acknowledgements. This work utilizes data obtained by the Global Oscillation Network Group (GONG) project, managed by the National Solar Observatory, a Division of the National Optical Astronomy Observatories, which is operated by AURA, Inc. under a cooperative agreement with the National Science Foundation. The data were acquired by instruments operated by the Big Bear Solar Observatory, High Altitude Obseratory, Learmonth Solar Observatory, Udaipur Solar Observatory, Instituto de Astrofisico de Canarias, and Cerro Tololo Interamerican Observatory. Our work is supported by the UK Particle Physics and Astronomy Research Council, through grant GR/J00588.

\section{References}

Abrams D., Kumar P., 1996, ApJ 472, 882

Anderson E., Duvall T., Jefferies S.M., 1990, ApJ 364, 699

Appourchaux T., Gizon L., Rabello-Soares M.-C., 1998, A\&A (in press)

Balmforth N.J., Gough D.O., 1990, Solar Phys. 128, 161

Bevington P.R., 1969, Data Reduction and Error Analysis in the Physical Sciences. McGraw Hill, New York, p. 238

Deubner F.-L., Gough D.O., 1984, ARA\&A 22, 593

Elsworth Y., Isaak I., Jefferies S.M., et al., 1988, in: Seismology of the Sun and Sun-Like Stars, Rolfe E. (ed.). ESA, Noordwijk, SP-286, p. 27

Harvey J., et al., 1996, Sci 272, 1284

Hill F., Stark P.B., Stebbins R.T., et al., 1996, Sci 272, 1292

Jefferies S.M., Duvall T.L., Harvey J.W., Osaki Y., Pomerantz M.A., 1991, ApJ 377, 330

Libbrecht K.G., 1988, ApJ 334, 510

Nigam R., Kosovichev A.G., Scherrer P.H., 1998, in: Poster Volume; Proc. IAU Symposium No. 181: Sounding Solar and Stellar Interiors, Nice, Sept. 30 - Oct. 3, 1996, Provost J. \& Schmider F.X. (eds.). Nice Observatory, p. 191

Press W.H., Flannery P.B., Teukolsky S.A., Vetterling T.A., 1989, Numerical Recipes (Fortran Version, First Edition). Cambridge University Press, Cambridge, p. 310

Schou J., Brown T.M., 1994, A\&AS 107, 541 